\title{
Derivation of energy equation for turbulent flow with two-point correlation
}

\author{
Shams Forruque Ahmed \\ Senior Lecturer in Mathematics, Prime University, Dhaka, Bangladesh
}

Email address:

Ahmed_jhe99@yahoo.com

To cite this article:

Shams Forruque Ahmed. Derivation of Energy Equation for Turbulent Flow with Two-Point Correlation. Pure and Applied Mathematics Journal. Vol. 2, No. 6, 2013, pp. 197-200. doi: 10.11648/j.pamj.20130206.15

\begin{abstract}
The energy equation for turbulent flow has been derived in terms of correlation tensors of second order, where the correlation tensors are the functions of space coordinates, distance between two points and time. An independent variable has been introduced in order to differentiate between the effects of distance and location. To reveal the relation of turbulent energy between two points, one point has been taken as the origin of the coordinate system. Correlation between pressure fluctuations and velocity fluctuations at the two points of flow field is applied to the turbulent energy equation.
\end{abstract}

Keywords: Energy Equation, Turbulent Flow, Two-Point Correlation, Correlation Tensor

\section{Introduction}

The turbulent flow can be found in many areas of industry, such as the production of the composite materials, environmental engineering, chemical engineering, textile industry, paper making and so on. The turbulence is maintained by the turbulent energy production, where the dissipation and the buoyancy flux act as sinks for the turbulent energy. Numerical models for turbulent fluid-particle flows were reviewed by Crowe et al. [1]. The review was structured according to the turbulence models used for the continuous phase: turbulence energy dissipation models, large-eddy simulations, direct numerical simulations, and discrete vortex models. Oakey [2] examined the rate of dissipation of turbulent energy from simultaneous temperature and velocity shear microstructure measurements. Spectra of turbulence were examined for both temperature gradient and velocity shear. Saito and Saito and Lemos [3] derived a macroscopic two-energy equation model for turbulent flow in a highly porous medium and applied to a porous channel bounded by parallel plates Macroscopic continuity, momentum and energy equations are presented local non-thermal equilibrium is considered by means of independent equations for the solid matrix and the working fluid.

Ozmidov [4] conferred the derivation of an eddy coefficient for the buoyancy flux on the foundation of a critical mixing distance. In the model of Ozmidov for oceanic turbulence there are mixing layers up to some vertical thickness determined by the stratification. Lilly et al. [5] estimated stratospheric mixing from high-altitude turbulence measurements. Nash and Moum [6] predicted microstructure of turbulent salinity flux and the dissipation spectrum of salinity. Williams and Gibson [7] measured the turbulence directly in the pacific equatorial undercurrent. Gargett [8] investigated the occurrence of oceanic turbulence with respect to fine structure. Osborn [9] recommended that the thick dissipation patches may be regions where the turbulence is supported by the Reynolds stress working against the local mean shear. This mean shear would be time variable, largely due to internal waves and hence would grow and decay with time. Weinstock [10] obtained vertical turbulent diffusion in a stably stratified fluid. Moum et al. [11] compared the turbulence kinetic energy dissipation rate estimates from two ocean microstructure profilers. Dillon and Caldwell [12] discussed the Batchelor spectrum and dissipation in the upper ocean.

Luketina and Imberger [13] determined the kinetic energy dissipation for turbulent flow from batchelor curve fitting. Moum [14] explored the energy-containing scales of turbulence in the ocean thermo cline. Sarker and Ahmed [15] conferred the motion of fibers in turbulent flow in a rotating system. Osborn [16] examined the energetic of the current and balanced the turbulent energy equation to justify using as an estimation of the local production. Hinze [17] derived an expression for turbulent motion with the correlation between pressure fluctuations and velocity 
fluctuations at two points of the flow field. Ahmed and Sarker [18] derived an equation for turbulent fiber motion in terms of second order correlation tensor, where the correlation tensors were the functions of space coordinates, distance between two points and time. In presence of dust particles, they derived another equation of turbulent fiber motion in terms of second order correlation tensor [19]. However, there are few studies relevant to the turbulent energy although it is prevalent in the industry. In view of all the work, the main aim of the present study is to derive energy equation of second order correlation tensor for turbulent flow with the aid of pressure-velocity correlation.

\section{Mathematical Model of the Problem}

The equations of motion and continuity for turbulent flow of a viscous incompressible fluid are

$$
\begin{aligned}
\frac{\partial u_{i}}{\partial t}+u_{j} \frac{\partial u_{i}}{\partial x_{j}} & =-\frac{1}{\rho} \frac{\partial p}{\partial x_{i}}+v \frac{\partial^{2} u_{i}}{\partial x_{j} \partial x_{j}} \\
\frac{\partial u_{i}}{\partial x_{i}} & =0
\end{aligned}
$$

The energy equation for turbulent flow of a viscous incompressible fluid is given by

$$
\frac{\partial u_{i}}{\partial t}+u_{j} \frac{\partial u_{i}}{\partial x_{j}}=-\frac{1}{\rho} \frac{\partial p}{\partial x_{i}}+v \frac{\partial^{2} u_{i}}{\partial x_{j} \partial x_{j}}-2 \varepsilon_{i j l} \Omega_{j} u_{l}
$$

where $u_{i}$ are the fluid velocity components; $p$ is the unknown pressure field; $v$ is the kinematical viscosity of the fluid; $\rho$ is the density of the fluid particle; $\varepsilon_{i j l}$ the three-dimensional permutation symbol, where $\mathcal{E}$ is the dissipation by turbulence per unit of mass; $\Omega_{i}$, the rotation vector and $t$ is the time.

We assume that the mean velocity $\bar{U}_{i}$ is constant throughout the region considered and independent of time, and we put

$$
\begin{aligned}
& \left(U_{i}=\bar{U}_{i}+u_{i}\right)_{A}, \\
& \left(U_{j}=\bar{U}_{j}+u_{j}\right)_{B} .
\end{aligned}
$$

The value of each term can be obtained by using the equations of motion for $u_{j}$ at the point $B$ and for $u_{i}$ at the point $A$. The energy equation for $u_{i}$ at the point $A$ is obtained from equation (3),

$$
\begin{aligned}
& \frac{\partial u_{i}}{\partial t}+\left(\bar{U}_{k}+u_{k}\right) \frac{\partial u_{i}}{\partial x_{k}}=-\frac{1}{\rho} \frac{\partial p}{\partial x_{i}} \\
& +v \frac{\partial^{2} u_{i}}{\partial x_{k} \partial x_{k}}-2 \varepsilon_{i k l} \Omega_{k} u_{l}
\end{aligned}
$$

For an incompressible fluid $\left(u_{i} \frac{\partial u_{k}}{\partial x_{k}}\right)_{A}=0$ so that equation (4) can be written as

$$
\begin{aligned}
& \frac{\partial}{\partial t}\left(u_{i}\right)_{A}+\left[\bar{U}_{k}+\left(u_{k}\right)_{A}\left(\frac{\partial}{\partial x_{k}}\right)_{A}\left(u_{i}\right)_{A}+\left(u_{i} \frac{\partial u_{k}}{\partial x_{k}}\right)_{A}\right. \\
& =-\frac{1}{\rho}\left(\frac{\partial}{\partial x_{i}}\right)_{A} p_{A}+\vartheta\left(\frac{\partial^{2}}{\partial x_{k} \partial x_{k}}\right)_{A}\left(u_{i}\right)_{A}-\left(2 \varepsilon_{i k l} \Omega_{k} u_{l}\right)_{A}
\end{aligned}
$$

Multiplying equation (5) by $\left(u_{j}\right)_{B}$ we obtain

$$
\begin{aligned}
& \left(u_{j}\right)_{B} \frac{\partial}{\partial t}\left(u_{i}\right)_{A}+\left[\bar{U}_{k}+\left(u_{k}\right)_{A}\left(\frac{\partial}{\partial x_{k}}\right)_{A}\left(u_{i}\right)_{A}\left(u_{j}\right)_{B}\right. \\
& +\left(u_{i}\right)_{A}\left(\frac{\partial}{\partial x_{k}}\right)_{A}\left(u_{k}\right)_{A}\left(u_{j}\right)_{B}=-\frac{1}{\rho}\left(\frac{\partial}{\partial x_{i}}\right)_{A} p_{A}\left(u_{j}\right)_{B} \\
& +v\left(\frac{\partial^{2}}{\partial x_{k} \partial x_{k}}\right)_{A}\left(u_{i}\right)_{A}\left(u_{j}\right)_{B}-\left(2 \varepsilon_{i k l} \Omega_{k} u_{l}\right)_{A}\left(u_{j}\right)_{B}
\end{aligned}
$$

Where $\left(u_{j}\right)_{B}$ can be treated as a constant in a differential process at the point $A$.

Similarly, the energy equation for $u_{j}$ at the point $B$ is obtained as

$$
\begin{aligned}
& \frac{\partial u_{j}}{\partial t}+\left(\bar{U}_{k}+u_{k}\right) \frac{\partial u_{j}}{\partial x_{k}}=-\frac{1}{\rho} \frac{\partial p}{\partial x_{j}} \\
& +v \frac{\partial^{2} u_{j}}{\partial x_{k} \partial x_{k}}-2 \varepsilon_{j k l} \Omega_{k} u_{l}
\end{aligned}
$$

For an incompressible fluid $\left(u_{j} \frac{\partial u_{k}}{\partial x_{k}}\right)_{B}=0$ so that equation (7) can be written as

$$
\begin{aligned}
& \frac{\partial}{\partial t}\left(u_{j}\right)_{B}+\left[\bar{U}_{k}+\left(u_{k}\right)_{B}\left(\frac{\partial}{\partial x_{k}}\right)_{B}\left(u_{j}\right)_{B}+\left(u_{j} \frac{\partial u_{k}}{\partial x_{k}}\right)_{B}\right. \\
& =-\frac{1}{\rho}\left(\frac{\partial}{\partial x_{j}}\right)_{B} p_{B}+v\left(\frac{\partial^{2}}{\partial x_{k} \partial x_{k}}\right)_{B}\left(u_{j}\right)_{B}-\left(2 \varepsilon_{j k l} \Omega_{k} u_{l}\right)_{B}
\end{aligned}
$$

Multiplying equation (8) by $\left(u_{i}\right)_{A}$, we get

$$
\begin{aligned}
& \left(u_{i}\right)_{A} \frac{\partial}{\partial t}\left(u_{j}\right)_{B}+\left[\bar{U}_{k}+\left(u_{k}\right)_{B}\left(\frac{\partial}{\partial x_{k}}\right)_{B}\left(u_{j}\right)_{B}\left(u_{i}\right)_{A}\right. \\
& +\left(u_{j}\right)_{B}\left(\frac{\partial}{\partial x_{k}}\right)_{B}\left(u_{k}\right)_{B}\left(u_{i}\right)_{A}=-\frac{1}{\rho}\left(\frac{\partial}{\partial x_{j}}\right)_{B} p_{B}\left(u_{i}\right)_{A} \\
& +v\left(\frac{\partial^{2}}{\partial x_{k} \partial x_{k}}\right)_{B}\left(u_{j}\right)_{B}\left(u_{i}\right)_{A}-\left(2 \varepsilon_{j k l} \Omega_{k} u_{l}\right)_{B}\left(u_{i}\right)_{A}
\end{aligned}
$$

Where $\left(u_{i}\right)_{A}$ can be treated as a constant in a differential process at the point $B$. 
Addition of equations (6) and (9) gives the result

$$
\begin{aligned}
& \frac{\partial}{\partial t}\left(u_{i}\right)_{A}\left(u_{j}\right)_{B}+\left[\left(\frac{\partial}{\partial x_{k}}\right)_{A}\left(u_{i}\right)_{A}\left(u_{k}\right)_{A}\left(u_{j}\right)_{B}+\left(\frac{\partial}{\partial x_{k}}\right)_{B}\left(u_{i}\right)_{A}\left(u_{k}\right)_{B}\left(u_{j}\right)_{B}\right] \\
& +\bar{U}_{k}\left[\left(\frac{\partial}{\partial x_{k}}\right)_{A}\left(u_{i}\right)_{A}\left(u_{j}\right)_{B}+\left(\frac{\partial}{\partial x_{k}}\right)_{B}\left(u_{i}\right)_{A}\left(u_{j}\right)_{B}\right]=-\frac{1}{\rho}\left[\left(\frac{\partial}{\partial x_{i}}\right)_{A} p_{A}\left(u_{j}\right)_{B}\right. \\
& \left.+\left(\frac{\partial}{\partial x_{j}}\right)_{B} p_{B}\left(u_{i}\right)_{A}\right]+v\left[\left(\frac{\partial^{2}}{\partial x_{k} \partial x_{k}}\right)_{A}+\left(\frac{\partial^{2}}{\partial x_{k} \partial x_{k}}\right)_{B}\right]\left(u_{i}\right)_{A}\left(u_{j}\right)_{B} \\
& -2\left[\left(\varepsilon_{i k} \Omega_{k} u_{l}\right)_{A}\left(u_{j}\right)_{B}+\left(\varepsilon_{j k l} \Omega_{k} u_{l}\right)_{B}\left(u_{i}\right)_{A}\right]
\end{aligned}
$$

To expose the relation of turbulent energy at the point $B$ to those at point $A$, it will give no difference if we take one point as the origin of $A$ or $B$ of the coordinate system.

Let us consider the point $A$ as the origin. In order to differentiate between the effects of distance and location, we introduce as new independent variables,

$$
\zeta_{k}=\left(x_{k}\right)_{B}-\left(x_{k}\right)_{A}
$$

Then we obtain,

$$
\begin{gathered}
\left(\frac{\partial}{\partial x_{k}}\right)_{A}=-\frac{\partial}{\partial \zeta_{k}},\left(\frac{\partial}{\partial x_{k}}\right)_{B}=\frac{\partial}{\partial \zeta_{k}} \\
\left(\frac{\partial^{2}}{\partial x_{k} \partial x_{k}}\right)_{A}=\left(\frac{\partial^{2}}{\partial x_{k} \partial x_{k}}\right)_{B}=\frac{\partial^{2}}{\partial \zeta_{k} \partial \zeta_{k}} .
\end{gathered}
$$

Using the above relations in equation (10) and taking ensemble average on both sides, equation (10) becomes

$$
\begin{aligned}
& \frac{\partial}{\partial t} \overline{\left(u_{i}\right)_{A}\left(u_{j}\right)_{B}}-\frac{\partial}{\partial \zeta_{k}} \overline{\left(u_{i}\right)_{A}\left(u_{k}\right)_{A}\left(u_{j}\right)_{B}}+\frac{\partial}{\partial \zeta_{k}} \overline{\left(u_{i}\right)_{A}\left(u_{k}\right)_{B}\left(u_{j}\right)_{B}} \\
& =-\frac{1}{\rho}\left[-\frac{\partial}{\partial \zeta_{i}} \overline{p_{A}\left(u_{j}\right)_{B}}+\frac{\partial}{\partial \zeta_{j}} \overline{p_{B}\left(u_{i}\right)_{A}}\right]+2 v \frac{\partial^{2}}{\partial \zeta_{k} \partial \zeta_{k}} \overline{\left(u_{i}\right)_{A}\left(u_{j}\right)_{B}} \\
& -2\left[\overline{\left(\varepsilon_{i k l} \Omega_{k} u_{l}\right)_{A}\left(u_{j}\right)_{B}}+\overline{\left(\varepsilon_{j k l} \Omega_{k} u_{l}\right)_{B}\left(u_{i}\right)_{A}}\right]
\end{aligned}
$$

Equation (11) represents the mean motion for turbulent energy with pressure-velocity correlation.

It is noted that the coefficient of $\bar{U}_{k}$ has been vanished. The equation (11) describes the turbulent energy motion, where the motions with respect to a coordinate system moving with the mean velocity $\bar{U}_{k}$. Equation (11) contains the double velocity correlation $\overline{\left(u_{i}\right)_{A}\left(u_{j}\right)_{B}}$, double correlations such as $\overline{p_{A}\left(u_{j}\right)_{B}}$, triple correlations such as $\left(u_{i}\right)_{A}\left(u_{k}\right)_{A}\left(u_{j}\right)_{B}$ where all the terms apart from one another. The correlations

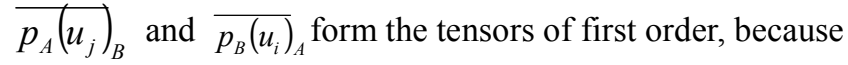
pressure is a scalar quantity and the triple correlations $\overline{\left(u_{i}\right)_{A}\left(u_{k}\right)_{A}\left(u_{j}\right)_{B}}$ and $\overline{\left(u_{i}\right)_{A}\left(u_{k}\right)_{B}\left(u_{j}\right)_{B}}$ form the tensors of third order.
We designate the first order correlations by $\left(k_{p, j}\right)_{A, B}$, second order correlations by $\left(Q_{i, j}\right)_{A, B}$ and third order correlations by $\left(s_{i k, j}\right)_{A, B}$.

Therefore, we set

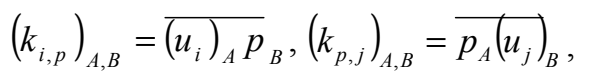

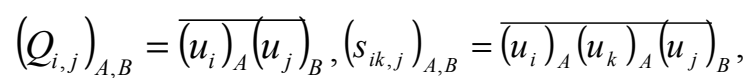

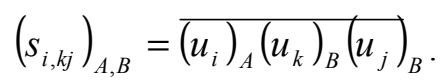

Where, the index $p$ indicates the pressure and is not a dummy index like $i$ or $j$ so that the summation convention does not apply to $p$.

Also the term $\overline{\left(\varepsilon_{i k l} \Omega_{k} u_{l}\right)_{A}\left(u_{j}\right)_{B}}$ and $\overline{\left(\varepsilon_{j k l} \Omega_{k} u_{l}\right)_{B}\left(u_{i}\right)_{A}}$ form the correlation tensors of second order, we designate these by $D_{i, j}$ and $H_{i, j}$ respectively. Thus we set

$$
\begin{aligned}
& \left(D_{i, j}\right)_{A, B}=\overline{\left(\varepsilon_{i k l} \Omega_{k} u_{l}\right)_{A}\left(u_{j}\right)_{B},} \\
& \left(H_{i, j}\right)_{A, B}=\left(\varepsilon_{j k l} \Omega_{k} u_{l}\right)_{B}\left(u_{i}\right)_{A}
\end{aligned}
$$

If we use the above relations of first, second and third order correlations in equation (11) then we obtain

$$
\begin{aligned}
& \frac{\partial}{\partial t} Q_{i, j}-\frac{\partial}{\partial \zeta_{k}} S_{i k, j}+\frac{\partial}{\partial \zeta_{k}} S_{i, k j}=-\frac{1}{\rho}\left(-\frac{\partial}{\partial \zeta_{i}} K_{p, j}+\frac{\partial}{\partial \zeta_{j}} K_{i, p}\right) \\
& +2 v \frac{\partial^{2}}{\partial \zeta_{k} \partial \zeta_{k}} Q_{i, j}-2\left[\left(D_{i, j}+H_{i, j}\right)\right]
\end{aligned}
$$

Where all the correlations refer to the two points $A$ and $B$.

Now for an isotropic turbulence of an incompressible flow, the double pressure-velocity correlations are zero, that is,

$$
\left(k_{p, j}\right)_{A, B}=0,\left(k_{i, p}\right)_{A, B}=0 .
$$

In an isotropic turbulence it follows from the condition of invariance under reflection with respect to point $A$,

$$
\overline{\left(u_{i}\right)_{A}\left(u_{k}\right)_{B}\left(u_{j}\right)_{B}}=\overline{-\left(u_{k}\right)_{A}\left(u_{j}\right)_{A}\left(u_{i}\right)_{B}}
$$

or, $\left(s_{i, k j}\right)_{A, B}=-\left(s_{k j, i}\right)_{A, B}$

Thus equation (12) can be written as

$$
\begin{aligned}
& \frac{\partial}{\partial t} Q_{i, j}-\frac{\partial}{\partial \zeta_{k}}\left(S_{i k, j}+S_{k j, i}\right) \\
& =2 v \frac{\partial^{2}}{\partial \zeta_{k} \partial \zeta_{k}} Q_{i, j}-2\left[\left(D_{i, j}+H_{i, j}\right)\right]
\end{aligned}
$$


The term $\frac{\partial}{\partial \zeta_{k}}\left(S_{i k, j}+S_{k j, i}\right)$ forms the tensor of second order; we designate these by $S_{i, j}$,

that is, $S_{i, j}=\frac{\partial}{\partial \zeta_{k}}\left(S_{i k, j}+S_{k j, i}\right)$.

Therefore equation (13) gives the result

$$
\frac{\partial}{\partial t} Q_{i, j}-S_{i, j}=2 v \frac{\partial^{2}}{\partial \zeta_{k} \partial \zeta_{k}} Q_{i, j}-2\left[\left(D_{i, j}+H_{i, j}\right)\right]
$$

This is the energy equation for turbulent flow in terms of correlation tensors of second order.

If there are no effects of the dissipation $\varepsilon$ by the turbulence per unit mass then $D_{i, j}=0, H_{i, j}=0$ so that the equation (14) takes the form

$$
\frac{\partial}{\partial t} Q_{i, j}-S_{i, j}=2 v \frac{\partial^{2}}{\partial \zeta_{k} \partial \zeta_{k}} Q_{i, j}
$$

This equation represents the turbulent motion in terms of correlation tensors of second order, which is the same as obtained by Hinze [4].

\section{Discussion and Conclusion}

The energy equation of second order correlation tensor for turbulent flow has been derived by averaging procedure, which consists of the correlations between pressure fluctuations and velocity fluctuations at the two points A and $\mathrm{B}$ of the flow field. The equation (14) demonstrates the energy equation of turbulent motion in terms of correlation tensors of second order. In the equation, all the terms $Q_{i, j}, S_{i, j}, D_{i, j}, H_{i, j}$ are the second order correlation tensors where, $Q_{i, j}$ and $S_{i, j}$ represents the velocity correlations at the two points $\mathrm{A}$ and $\mathrm{B}$ of the flow field; $D_{i, j}$ and $H_{i, j}$ are the velocity correlations for turbulent energy. But in absence of the dissipation $\varepsilon$ by turbulence per unit of mass, the resulting equation (14) reduces to the equation (15) which confers the turbulent motion in terms of correlation tensors of second order.

\section{References}

[1] C.T. Crowe, T.R. Troutt and J.N. Chung, "Numerical models for two-phase turbulent flows", Annual Review of Fluid Mechanics, vol. 28, pp.11-43, 1996.

[2] N.S. Oakey, "Determination of the rate of dissipation of turbulent energy from simultaneous temperature and velocity shear microstructure measurements", J. Phys. Oceanogr., Vol. 12, pp. 256-271, 1982.

[3] M.B. Saito and M.J.S. de Lemos, "A macroscopic two-energy equation model for turbulent flow and heat transfer in highly porous media", vol. 35 , pp. 2424-2433, 2010 .

[4] R.V. Ozmidov, "On the turbulent exchange in a stably stratified ocean, Atmos", Ocean Phys., vol. 8, pp. 853-860, 1965.

[5] D.K. Lilly, D.E. Wacoand and S.I. Adelfang, "Stratospheric mixing estimated from high-altitude turbulence measurements", J. Appl. Meteor., vol. 131, pp. 488-493, 1974.

[6] J.D. Nash and J. N. Moum, "Microstructure Estimates of Turbulent Salinity Flux and the Dissipation Spectrum of Salinity”, J. Phys. Oceanogr., vol. 32, pp. 2312-2333, 2002.

[7] R.B. Williams and C.H. Gibson, "Direct measurements of turbulence in the pacific equatorial undercurrent", J. Phys. Oceanogr., vol. 4, pp. 104-108, 1974.

[8] A.E. Gargett, "An investigation of the occurrence of oceanic turbulence with respect to fine structure", J. Phys. Oceanogr., vol. 6 , pp. 139-156, 1976.

[9] T.R. Osborn, "Estimates of the Local Rate of Vertical Diffusion from Dissipation Measurements", J. Phys. Oceanogr., vol. 10, pp. 83-89, 1980.

[10] J. Weinstock, "Vertical turbulent diffusion in a stably stratified fluid", J. Atmos. Sci., vol. 35, pp. 1022-1027, 1978.

[11] J.N. Moum, M.C. Gregg, R.C. Lien and M.E. Carr, "Comparison of turbulence kinetic energy dissipation rate estimates from two ocean microstructure profilers", J. Atmos. Oceanic Technol., vol. 12, pp. 346-366, 1995.

[12] T.M. Dillon and D.R. Caldwell, "The Batchelor Spectrum and Dissipation in the Upper Ocean", J. Geophys. Res., vol. 85, no. C4, pp. 1910-1916, 1980.

[13] D.A. Luketina and J. Imberger, "Determining turbulent kinetic energy dissipation from batchelor curve fitting", J. Atmos. Oceanic Technol., vol. 18, pp. 100-113, 2001.

[14] J.N. Moum, Energy-containing scales of turbulence in the ocean thermo cline. J. Geophys. Res., vol. 101, no. C6, pp.14095-14109, 1996b.

[15] M.S.A. Sarker and S.F. Ahmed, "Motion of Fibers in Turbulent Flow in a Rotating System", Rajshahi University Studies Part-B, Journal of Science, vol. 37, pp. 107-117, 2009.

[16] T.R. Osborn, Measurements of energy dissipation adjacent to an island, J.Geophys. Res., vol. 83, pp. 2939-2957, 1978.

[17] J. O. Hinze, Turbulence, McGraw-Hill Book Co., New York, 1959.

[18] S.F. Ahmed and M.S.A. Sarker, "Fiber suspensions in turbulent flow with two-point correlation. Bangladesh Journal of Scientific and Industrial Research", vol. 46, no. 2, pp. 265-270, 2011.

[19] M.S.A. Sarker and S.F. Ahmed, "Fiber motion in dusty fluid turbulent flow with two-point correlation", Journal of Scientific Research, vol. 3, no. 2, pp. 283-290, 2011. 